\title{
A THEOREM IN THE REPRESENTATION THEORY OF TOTALLY DISCONNECTED GROUPS ${ }^{1}$
}

\author{
ROBERT R. KALLMAN
}

\begin{abstract}
We prove that certain von Neumann algebras naturally associated with specific direct summands of the left regular representation of a unimodular totally disconnected group are finite von Neumann algebras. The Lie group analogue of this result is false.
\end{abstract}

The purpose of this note is to prove the following theorem. It probably will be useful in analyzing the left regular representation of a unimodular totally disconnected group.

Theorem 1. Let $G$ be a unimodular locally compact group whose topology has a countable basis, and let $K$ be a compact open subgroup of $G$. Let $\pi$ be an irreducible unitary representation of $K$, and let $U^{\pi}$ be the unitary representation of $G$ induced by $\pi$. Then $\left(U^{\pi}\right)^{\prime}$, the commutant of $U^{\pi}$, is a finite von Neumann algebra, and there is an explicit formula for a faithful, finite, normal trace on $\left(U^{\pi}\right)^{\prime}$.

An immediate corollary is the following.

Corollary 2. Let $G$ and $K$ be as in Theorem 1 , and let $\pi$ be a unitary representation of $K$ which is a finite direct sum of irreducibles. Then $\left(U^{\pi}\right)^{\prime}$ is a finite von Neumann algebra.

Note that the most plausible Lie group analogue of Theorem 1 is false. Let $H$ be a connected Lie group whose Lie algebra is a Heisenberg Lie algebra and whose center, $C$, is compact. $H$ is unimodular and $C$ is a maximal compact normal subgroup of $H$. Let $\chi$ be a nontrivial character of $C$. Then $U^{\boldsymbol{\chi}}$ is a central direct summand of the left regular representation of $H$, which is an infinite multiple of a fixed infinite dimensional irreducible unitary representation of $H$ by the Stone-von Neumann theorem. Hence, $\left(U^{\boldsymbol{x}}\right)^{\prime}$ is not a finite von Neumann algebra.

Received by the editors September 10, 1973.

AMS (MOS) subject classifications (1970). Primary 22D10.

Key words and phrases. Unitary representations of locally compact groups, von Neumann algebras.

1 Supported in part by NSF Grant GP-38023. 
We first recall some basic results of G. W. Mackey [1]. Let $G$ and $K$ be as in Theorem 1. Let $\pi$ be a unitary representation of $K$ on the Hilbert space $\mathbf{H}(\pi)$. Recall how $U^{\pi}$ is defined. $\mathbf{H}\left(U^{\pi}\right)=[f: G \rightarrow \mathbf{H}(\pi) \mid f(k g)=$ $\pi(k) f(g), k \in K, g \in G]$ with the inner product $\left(f_{1}, f_{2}\right)=\Sigma_{g \in K \backslash G}\left(f_{1}(g), f_{2}(g)\right)$. Notice that this sum is countable since $K \backslash G$ is countable. One then defines $\left(U^{\pi}(g) f\right)(a)=f(a g)$, and one checks that $U^{\pi}(g)$ is a unitary representation of $G$ on $\mathbf{H}\left(U^{\pi}\right)$ and that $g \rightarrow U^{\pi}(g)$ is strongly continuous. Fix $y$ in $G$ and $v$ in $\mathbf{H}(\pi)$. Define $f(y, v, \cdot)$ in $\mathbf{H}\left(U^{\pi}\right)$ by setting $f(y, v, x)=0$ if $x$ is not an element of $K \cdot y$ and $f(y, v, k \cdot y)=\pi(k)(v)$. Notice that any element of $\mathbf{H}\left(U^{\pi}\right)$ which vanishes outside of $K \cdot y$ has the form $f(y, v, \cdot)$ for some $y$ in $G$ and $v$ in $\mathbf{H}(\pi)$. Every element of $\mathbf{H}\left(U^{\pi}\right)$ is a unique sum over $K \backslash G$ of elements of $\mathbf{H}\left(U^{\pi}\right)$ which vanish outside of these cosets. Let $T$ be a bounded linear operator on $\mathbf{H}\left(U^{\pi}\right)$. $T$ is completely determined by its values on the $f(y, v, \cdot)$. Set $T(x, y)(v)=(T(f(y, v, \cdot)))(x) . T(x, y)$ is an element of $L(\mathbf{H}(\pi))$, for $\|T(x, y)(v)\| \leq\|T\| \cdot\|f(y, v, \cdot)\|=\|T\| \cdot\|v\|$. One easily checks that:

(A) $T\left(k_{1} x, k_{2} y\right)=\pi\left(k_{1}\right) T(x, y) \pi\left(k_{2}\right)^{*}$;

(B) $T^{*}(x, y)=T(y, x)^{*}$;

(C) for each $f$ in $\mathbf{H}\left(U^{\pi}\right)$,

$$
(T(f))(x)=\sum_{y \in K \backslash G} T(x, y)(f(y)) ;
$$

(D) for each $f$ in $\mathbf{H}\left(U^{\pi}\right)$,

$$
\left(T^{*}(f)\right)(x)=\sum_{y \in K \backslash \sigma .} T(y, x)^{*}(f(y)) ;
$$

(E) $T$ commutes with $U^{\pi}$ if and only if $T(x g, y g)=T(x, y)$ for all $x, y$, and $g$ in $G$;

(F) $(S T)(x, y)=\Sigma_{a \in K \backslash G} S(x, a) T(a, y)$, the summation converging in the strong operator topology independent of order and the $a$ ranging over distinct $K \backslash G$ coset representatives.

Before going on with the proof of Theorem 1, we need to prove the following simple but crucial lemma. It is the only place where we use the hypothesis that $G$ is unimodular. If $a$ is in $G$, set $K(a)=a^{-1} K a$. If $a_{1}, \cdots$, $a_{n}$ are elements of $G$, set $K\left(a_{1}, \cdots, a_{n}\right)=K\left(a_{1}\right) \cap \cdots \cap K\left(a_{n}\right)$. Note that $K\left(a_{1}, \cdots, a_{n}\right)$ is a compact open subgroup of $G$. Hence, $K\left(a_{1}\right) / K\left(a_{1}, \cdots, a_{n}\right)$ is finite. Let $\#\left(K\left(a_{1}\right) / K\left(a_{1}, \cdots, a_{n}\right)\right)$ be the number of elements in this quotient space. 
Lemma 3. Let $a$ and $b$ be arbitrary elements of $G$. Then

$$
\#(K(a) / K(a, b))=\#(K(b) / K(a, b)) .
$$

Proof. Let $\mu$ be Haar measure on $G$, normalized so that $\mu(K)=1$. Note that $\#(K(a) / K(a, b))=\#\left(K / K\left(e, b a^{-1}\right)\right)$ and $\#(K(b) / K(a, b))=\#\left(K / K\left(e, a b^{-1}\right)\right)$. But if $K^{\prime}$ is an open subgroup of $K$, then $\#\left(K / K^{\prime}\right)=\mu\left(K^{\prime}\right)^{-1}$. Hence, it suffices to show that for any $z$ in $G, \mu(K(e, z))=\mu\left(K\left(e, z^{-1}\right)\right)$. But this is true, for $G$ is unimodular, and $K(e, z)=z^{-1} K\left(e, z^{-1}\right) z$. Q.E.D.

We now prove Theorem 1. First of all note that if $T$ is an element of $\left(U^{\pi}\right)^{\prime}$, then $T(e, e)$ is a scalar, for $\pi(k) T(e, e) \pi(k)^{*}=T(k, k)=T(e, e)$, by (A) and (E), and $\pi$ is irreducible. One easily checks that $T \rightarrow \rho(T)=$ $T(e, e)$ is linear, $\rho(I)=1$, and $\rho(T) \geq 0$ if $T \geq 0$. We claim that $\rho(T)$ is a faithful normal finite trace on $\left(U^{\pi}\right)^{\prime}, \rho(T)$ is clearly normal. $\rho(T)$ is faithful, for $\rho(T)=0$ if and only if $T(e, e)=0$ if and only if $(T x, x)=0$, where $x=f(e, v, \cdot)$ for any (and every) nonzero $v$ in $\mathbf{H}(\pi)$. Hence, if $T \geq 0$, then $T^{1 / 2} x=0$. But $T$ and $U^{\pi}$ commute, $U^{\pi}(a)(f(e, v, \cdot))=f\left(a^{-1}, v, \cdot\right)$, and elements of $\mathbf{H}\left(U^{\pi}\right)$ of the form $f(b, v, \cdot)$, where $b$ is in $G$ and $v$ in $\mathbf{H}(\pi)$, have a linear span which is dense in $\mathbf{H}\left(U^{\pi}\right)$. Hence, $T^{1 / 2}=0$, and thus $T=0$. Hence, $T \rightarrow \rho(T)$ is faithful. To prove that $T \rightarrow \rho(T)$ is a trace on $\left(U^{\pi}\right)^{\prime}$, it suffices to show that $\rho\left(T T^{*}\right)=\rho\left(T^{*} T\right)$ for all $T$ in $\left(U^{\pi}\right)^{\prime}$. Let $(G \times G)_{d}$ be the diagonal subgroup of $G \times G$. Then the number of elements of $G \times G$ of the form $(e, b)$ which are in the same $(K \times K) \backslash(G \times G) /(G \times G)_{d}$ double coset as $(e, a)$, and such that the $b$ 's are in distinct $K \backslash G$ double cosets, is \#(K/K(e,a)). This follows easily by noting that $K \cdot g=K$ if and only if $g$ is in $K$, and $(K \cdot g, K a \cdot g)=(K, K a)$ if and only if $g$ is an element of $K \cap K(a)$. Let trace $(\cdot)$ be the trace on $L(H(\pi))$, normalized so that $\operatorname{trace}(I)=1$. This makes sense because $\mathbf{H}(\pi)$ is finite dimensional, for $K$ is compact and $\pi$ is irreducible. Notice that if $T$ is in $L\left(H\left(U^{\pi}\right)\right), T^{*}(e, a) T(a, e)$ is in $L(\mathbf{H}(\pi))$.

$$
\rho\left(T^{*} T\right)=\left(T^{*} T\right)(e, e)=\operatorname{trace}\left(\left(T^{*} T\right)(e, e)\right)=\sum_{a \in K \backslash G} \operatorname{trace}\left(T^{*}(e, a) T(a, e)\right),
$$

for the series $\Sigma_{a \in K \backslash G} T^{*}(e, a) T(a, e)$ converges in almost any reasonable topology since $L(\mathbf{H}(\pi))$ is finite dimensional. Now the number of $(e, b)$ which are in the same $(K \times K) \backslash(G \times G) /(G \times G)_{d}$ double coset as $(e, a)$ is $\#(K / K(e, a))$. Choose $b$ such that $(e, a)$ and $(e, b)$ are in the same $(K \times K) \backslash(G \times G) /(G \times G)_{d}$ double coset. Then $(e, b)=\left(k_{1} g, k_{2} a g\right)$ for some $k_{1}, k_{2}$ in $K$ and $g$ in $G$. Therefore 


$$
\begin{aligned}
\operatorname{trace}\left(T^{*}(e, b) T(b, e)\right) & =\operatorname{trace}\left(T^{*}\left(k_{1} g, k_{2} a g\right) T\left(k_{2} a g, k_{1} g\right)\right) \\
& =\operatorname{trace}\left(\pi^{*}\left(k_{1}\right) T^{*}(e, a) \pi\left(k_{2}\right)^{*} \pi_{2}\left(k_{2}\right) T(a, e) \pi\left(k_{1}\right)^{*}\right) \\
& =\operatorname{trace}\left(T^{*}(e, a) T(a, e)\right)
\end{aligned}
$$

by $(E)$. Hence,

$$
\begin{aligned}
& o\left(T^{*} T\right)=\sum_{D \in(K \times K) \backslash(G \times G) /(G \times G)} \#(K / K(e, a)) \operatorname{trace}\left(T^{*}(e, a) T(a, e)\right) \\
& (e, a) \in D \\
& =\sum_{D \in(K \times K) \backslash(G \times G) /(G \times G)_{d}} \#\left(K / K\left(e, a^{-1}\right)\right) \operatorname{trace}\left(T\left(e, a^{-1}\right) T^{*}\left(a^{-1}, e\right)\right) \\
& \left(a^{-1}, e\right) \in D
\end{aligned}
$$

by Lemma 3 and (E). Now as before, if $(p, e)$ and $(q, e)$ are in the same $(K \times K) \backslash(G \times G) /(G \times G)_{d}$ double coset, then trace $\left(T(e, p) T^{*}(p, e)\right)=$ $\operatorname{trace}\left(T(e, q) T^{*}(q, e)\right)$. Furthermore, as before, the number of $(p, e)$ which are in the same $(K \times K) \backslash(G \times G) /(G \times G)_{d}$ double coset as $(q, e)$, and such that the $p$ 's are in distinct $K \backslash G$ cosets, is \# $(K / K(e, q))$. Hence,

$$
\begin{aligned}
\rho\left(T^{*} T\right) & =\sum_{a \in K \backslash G} \operatorname{trace}\left(T(e, a) T^{*}(a, e)\right)=\operatorname{trace}\left(\sum_{a \in K \backslash G} T(e, a) T^{*}(a, e)\right) \\
& =\operatorname{trace}\left(\left(T T^{*}\right)(e, e)\right)=\left(T T^{*}\right)(e, e)=\rho\left(T T^{*}\right) . \quad \text { Q.E.D. }
\end{aligned}
$$

\section{BIBLIOGRAPHY}

1. G. W. Mackey, On induced representations of groups, Amer. J. Math. 73 (1951), 576-592. MR 13, 106.

DE PARTMENT OF MATHEMATICS, UNIVERSITY OF F LORIDA, GAINESVILLE, FLORIDA 32601 